\title{
The economic impact of cartels and anti-cartel enforcement
}

\author{
Stephen Davies \\ School of Economics, University of East Anglia \\ Peter L. Ormosi \\ Norwich Business School, University of East Anglia \\ ESRC Centre for Competition Policy \\ University of East Anglia \\ CCP Working Paper 13-7 v2 (2014)
}

\begin{abstract}
Evaluations of the consumer harm caused by cartels are typically partial because they do not attempt to quantify the impact of deterrence, or acknowledge that the CA does not root out all anti-competitive cases. This paper proposes a broader framework for evaluation which encompasses these unobserved impacts. Calibration of this framework is challenging because one cannot rely on estimates for cases which have been observed to make deductions about those that have not - an example of the classic sample selection problem which is endemic across much of the empirical Industrial Organisation literature. However, we show how empirical findings, already available in the existing literature, can be plugged into a Monte Carlo experiment to establish bound estimates on the magnitudes of cartel-induced consumer harm. Lower bound (i.e. cautious) estimates suggest that (i) the harm detected by the CA really is only the tip of the iceberg, accounting for only a small fraction (at most one sixth) of total potential harm; (ii) deterrence is at least twice as effective as detection as a means for removing harm; and (iii) undetected harm is at least twice as large as detected harm. Under less cautious, but very plausible, assumptions, all three effects could be much greater than this.
\end{abstract}

Keywords: cartels, anti-competitive harm, deterrence, detection, selection bias, Monte Carlo simulation JEL Classification codes: K21, L44, H11

Contact Details:

Stephen Davies

s.w.davies@uea.ac.uk

Peter Ormosi

p.ormosi@uea.ac.uk 


\title{
The economic impact of cartels and anti-cartel enforcement*
}

\author{
Stephen Davies ${ }^{\dagger}$ and Peter L. Ormosi ${ }^{\ddagger}$
}

November 8, 2014

\footnotetext{
*This paper is a significantly rewritten version of the paper 'Selection bias in evaluating policy: the case of anti-trust CCP', CCP WP 13-7. We are grateful for useful comments on earlier versions of this paper from Iwan Bos, Denis Carlton, Joe Harrington, Morten Hviid, Greg Werden, the editor and referees of this journal, and the participants of conferences organised by CCP (June 2013), LEAR (June 2013), ABA (June 2013), CRESSE (July 2014). The usual disclaimer applies. The support of the Economic and Social Research Council and the ESRC Centre for Competition Policy is gratefully acknowledged.

${ }^{\dagger}$ School of Economics and ESRC Centre for Competition Policy, University of East Anglia, Norwich Research Park, NR4 7TJ, Norwich, United Kingdom, email: s.w.davies@uea.ac.uk

${ }^{\ddagger}$ Norwich Business School and ESRC Centre for Competition Policy, University of East Anglia, Norwich Research Park, NR4 7TJ, Norwich, United Kingdom, email: p.ormosi@uea.ac.uk
} 


\begin{abstract}
Evaluations of the consumer harm caused by cartels are typically partial because they do not attempt to quantify the impact of deterrence, or acknowledge that the CA does not root out all anti-competitive cases. This paper proposes a broader framework for evaluation which encompasses these unobserved impacts. Calibration of this framework is challenging because one cannot rely on estimates for cases which have been observed to make deductions about those that have not - an example of the classic sample selection problem which is endemic across much of the empirical Industrial Organisation literature. However, we show how empirical findings, already available in the existing literature, can be plugged into a Monte Carlo experiment to establish bound estimates on the magnitudes of cartel-induced consumer harm. Lower bound (i.e. cautious) estimates suggest that (i) the harm detected by the CA really is only the tip of the iceberg, accounting for only a small fraction (at most one sixth) of total potential harm; (ii) deterrence is at least twice as effective as detection as a means for removing harm; and (iii) undetected harm is at least twice as large as detected harm. Under less cautious, but very plausible, assumptions, all three effects could be much greater than this.
\end{abstract}

Keywords: cartels, anti-competitive harm, deterrence, detection, selection bias, Monte Carlo simulation

JEL Classification codes: K21, L44, H11 


\section{Introduction}

With the growth of anti-cartel enforcement throughout the world, increasing efforts are now devoted to estimating its impact on economic welfare. Although there are some dissonant voices, ${ }^{1}$ most evaluations are very positive: estimates from various CAs, although fairly rough and ready, suggest that the benefits to consumers from their activities more than outweigh their cost, usually by an order of magnitude. For example, the European Commission (EC) estimates that in 2010 consumers benefited from the removal of overcharges by cartels that were detected and prohibited to the tune of $€ 7.2$ billion.

However such studies quantifying the consumer harm caused by cartels are based only on the cases that the CA busts - we call this the 'observed harm'. This raises the obvious question: what about the harm that goes unobserved, because it is deterred? After all a major function of any law is to deter antisocial behaviour, and this is hopefully also true for competition policy. It is often suggested that the magnitude of deterred harm far exceeds the harm removed by direct intervention, ${ }^{2}$ but the measurement of this effect poses significant challenges. It also raises a second question: what about a second type of unobserved case - those which the CA fails to detect, even though they involve anti-competitive harm? In effect, these represent a foregone opportunity, or even failure of policy; again, this is never quantified, although the magnitudes involved also could be substantial.

This suggests that a more encompassing approach would be to ask first "how much potential for anti-competitive harm is there out there in an economy?" and then to assess the success of policy by asking "how much of that potential harm is avoided and/or removed by the presence of anti-cartel laws and the activities of competition authorities?" The first question is reminiscent of an old literature, provoked by Harberger (1954) which attempted to quantify the "social costs of monopoly", although our approach is very different. Such an approach is undoubtedly ambitious and may even seem speculative, but the potential insights are far wider reaching

\footnotetext{
${ }^{1}$ For example, Crandall and Winston (2003) stridently reject any positive impact of competition policy, but Baker (2003) in the same journal issue paints a far more positive picture.

${ }^{2}$ See for example Werden (2008), Geroski (2004, p.3) and Baker (2003, p.40).
} 
than merely asking whether policy generates benefits which exceed its costs.

The purpose of this paper is to explore what we can establish from the characteristics of observed harm about the contemporaneous magnitude of total potential harm. It presents a conceptual framework, which marshals what we do know about this harm from a large database of cartel overcharges. The findings of previous robust empirical results are plugged into this framework, and a Monte Carlo experiment assists the calibrations to deduce the magnitudes of what we do not know - total, deterred and undetected harm. The paper provides some lower bound estimates that deterrence is at least twice as effective as detection as a means for removing harm, and undetected harm is at least twice as large as detected harm. Under less cautious, but very plausible, assumptions, total potential anticompetitive harm from cartels is 13.7 larger than the harm detected, deterred harm is nearly 10 times higher and undetected harm over 3 times greater than the CA's detected harm.

\subsection{Previous literature}

Existing evaluations of the impact of cartels have taken a variety of forms. Quantitative methods vary widely, including difference-in-differences, event studies, and surveys. ${ }^{3}$ However, all these works are constrained by the same limitation: driven mostly by data availability, inferences are made on the basis only of the sample of cases in which the CA has intervened, and fail to take account of unobserved cases, cases that are deterred or are undetected. Authors typically defend this approach by claiming reasonably that there is very little information on detection and deterrence. We will show that there is sufficient information to draw valuable insights on the unobserved parts of cartel harm distribution.

There are important empirical contributions in the wider literatures on cartel detection. The early work of Bryant and Eckard (1991) suggested that, in a given year, only $13-17 \%$ of cartels are detected, and Combe et al. (2008) confirm a similar magnitude for Europe using the same method. ${ }^{4}$ Ormosi (2014) proposes an alter-

\footnotetext{
${ }^{3}$ See Ormosi (2012) and Davies and Ormosi (2012) for summaries of these various literatures.

${ }^{4}$ Lande and Connor (2012) provides an exhaustive survey, including estimates derived from surveys.
} 
native method that draws an analogy with capture-recapture analysis in ecological science and finds detection rates to be in the same ballpark. However, these approaches relate to cartels which are eventually caught, and leave open the question of how many and what types of cartels are never observed because they are deterred or never detected.

The deterrent effect of law has been the subject of academic attention since the late 60s, with Becker (1968) paving the way for decades of law and economics research. More specifically on cartels, previous works have focused on different aspects of deterrence, such as the optimal amount of criminal sanctions, i.e. sanctions that help achieve highest deterrence, ${ }^{5}$ or whether revenue or profit should be used as a basis for calculating the optimal deterrent fine. ${ }^{6}$ More recently research turned to experiments, attempting a direct measurement of deterrence in a controlled laboratory environment. ${ }^{7}$

Relatively few works have provided empirical (out-of-lab) solutions for determining the level of cartel deterrence. Focusing specifically on the impact of leniency programmes, Miller (2009) offers a model of cartel formation over time and shows that the introduction of the 1993 leniency programme strengthened deterrence. In terms of examining the level of deterrence, competition authorities (CA) have relied on survey studies involving interviews of competition practitioners, lawyers and companies. In one such study, commissioned by the OFT in the UK, Deloitte (2007) reports that for each cartel detected by the $\mathrm{CA}$, there were at least another five that were deterred by competition law. ${ }^{8}$

At first sight, these detection and deterrence rate estimates provide a straightforward approach for extrapolating the estimates from CAs of how much harm they rectify from their detected cases to estimate the magnitudes of the unknown deterred

\footnotetext{
${ }^{5}$ See for example Elzinga and Breit (1973), Landes (1983), Kobayashi (2001), Ginsburg and Wright (2010), Werden et al. (2012).

${ }^{6}$ Katsoulacos and Ulph (2013).

${ }^{7}$ For a general experimental treatment of deterrence see Schildberg-Hörisch and Strassmair (2012). For cartels specifically see Bigoni et al. (2012).

${ }^{8}$ Subsequent studies using similar survey methodologies confirm that such multipliers are likely to be significant, although the precise magnitudes vary considerably: a larger follow-up UK survey by London Economics (2011) reports much higher (1:28) deterrence rates.
} 
or undetected harms. If for every case detected, it is known that another five are deterred, the magnitude of deterred harm might be inferred to be five times greater than the CA's estimates of the harm caused by the cartels it busts. Equally, with estimated detection rates of 1 in 6 for cartels, the magnitudes of undetected harm may be similarly estimated. We refer to this below as the simple multiplier approach, and argue that it confronts a strong possibility of selection bias - the sample observed (by the CA) may not be representative of the full population. ${ }^{9}$

Thus, this paper also confronts a sample selection problem, and is in that sense of wider relevance than just the narrowly defined policy evaluation literature. There is a significant body of empirical research in Industrial Organisation which employs datasets of cases investigated and intervened by CAs, and such data have provided a rich source of information on cartels (typical overcharge, duration, structural characteristics etc.). While many studies in that literature acknowledge the possibility of sample selection bias, this is rarely quantified, and it is unknown how far this might compromise many of the conventional wisdoms, for example, on cartel duration and overcharge.

\section{$1.2 \quad$ Structure}

Section 2 defines terms and introduces a framework which relates detected harm to an underlying population of all potential anti-competitive harm. It introduces how simple multipliers of the type just described could be used to provide estimates of the magnitudes of potential total population harm, deterred and undetected harms. The remainder of the paper explores sample selection bias in this setting. Section 3 frames the task as a simple sampling problem. Section 4 examines the likely properties of the samples of cartel harm which are observed in CA interventions. Section 5 uses Monte Carlo experiments based on information from previous empirical works and

\footnotetext{
${ }^{9}$ Of course, such an approach can also be criticised for attaching over-reliance to survey results, based on subjective assessments of sometimes hypothetical questions. Moreover, the ratios reported in survey estimates also include composition deterrence (when firms modify their conduct to avoid prosecution), where the amount of modification is likely to vary significantly across (potential) cartels.
} 
discusses the main findings and policy implications.

\section{Framework}

\section{$2.1 \quad$ Notation}

Denote the total potential cartel-induced harm in a given economy for a given point in time (say year) by $H$. This includes all harm that would happen in the absence of competition policy. ${ }^{10}$ Some of this harm does not occur because it is deterred by the existence of competition law and enforcement. Denote the magnitude of all

deterred harm by $W$ and its ratio to all harm by $\omega:=\frac{W}{H}$. If the conduct is not fully deterred then some/all of the undeterred harm is discovered by the CA. Denote the magnitude of all detected harm by $S$, and its ratio to all undeterred harm by $\sigma:=\frac{S}{H-W} \cdot{ }^{11}$

In general, both $\sigma$ and $\omega$ may be time variant and interdependent. This is not to deny the possibility of a dynamic causal relation running from $\omega$ to future $\sigma$ : success in detection by the $\mathrm{CA}$ might deter firms from attempting on contravene the law in the future. But the purpose of this paper is not to model such a relationship, rather to expose what we can establish from the characteristics of observed harm at a given time on the characteristics of the population harm at the same time.

The ratio of detected harm (i.e. undeterred and detected and therefore observed/sampled) to total population harm is given by $(1-\omega) \sigma$, and the magnitude of detected (sampled) harm is:

$$
H^{S}=(1-\omega) \sigma H
$$

\footnotetext{
${ }^{10}$ This counterfactual is over-simplification to the extent that private enforcement might anyway deter some such cases. It also abstracts from the possibility that other policy, such as import liberalisation or deregulation, might also deter behaviour. Finally, we acknowledge the possibility of harmless horizontal agreements but our focus is on harmful cases only. Although the possibility of a cartel with negative harm is very small, we address this issue in the Appendix.

${ }^{11}$ We assume that the CA does not make errors: a detected case is always rightly convicted. Although only a remote possibility in cartel cases, in Appendix B we discuss the possibility of Type 2 errors by the CA.
} 
In passing, note that Eq.(1) formalises that small detected harm does not necessarily imply a lazy or inept CA, it might also signal one, which is particularly effective in deterring - something that deserves emphasis in any policy evaluation exercise. Equally, a CA eliminating huge amounts of cartel harm may be one which is ineffectual in deterring.

\subsection{The simple multiplier}

Consider the implications for the magnitudes of total potential harm. Given that the $\mathrm{CA}$ is able to estimate the magnitude of harm it removes in the cases it detects and intervenes, this suggests a simple method for approximately estimating total population harm, and its unknown constituents: deterred and undetected harms.

Remark 1 If the harm detected and remedied by the $C A\left(H^{S}\right)$ is a random sample of the population, then total population harm is $H^{S} /((1-\omega) \sigma)$, of which $H^{S} \omega /((1-\omega) \sigma)$ is deterred and $H^{S}(1-\sigma) / \sigma$ is undetected. Hereafter, these are referred to as the "simple multiplier" estimates.

The main purpose of this paper is to explore how reasonable is the assumption of a random sample, how sensitive are estimates of the magnitudes of harm when the randomness assumption is relaxed, and how to derive estimates that account for this selection problem. Viewed in this way, the paper addresses a classic potential problem of selection bias and, as mentioned above, has wider implications for any empirical research in IO, where conclusions are drawn from databases based on observed cartels which are intervened by CAs. ${ }^{12}$

\footnotetext{
${ }^{12}$ For example, conventional wisdoms often drawn from the empirical literature are that, typically, cartels last for about 7 years, involve about 7 members and overcharge in the region of 15-30\%. All of these estimates are based on cartels which have been detected: little is known about those which are never detected, or those which are deterred.
} 


\section{Selection bias with differential sampling}

We now consider a sampling problem in which the purpose is to estimate the magnitude of total harm for the population $(H)$, from which a sample has been taken; the magnitude of sample harm $\left(H^{S}\right)$ and proportionate sample size (i.e. undeterred harm which is detected), $\lambda:=(1-\omega) \sigma$, are both known. In these terms, the above "simple multiplier" approach uses $\left(H^{S} / \lambda\right)$ as an estimator of $(H)$.

As we will discuss below, there are good reasons to believe that both $\omega$ and $\sigma$ (and therefore $\lambda$ ) are dependent on the level of harm $h$ and that therefore the simple multiplier will provide biased estimates. We show this using a stylised trichotomy, in which the population is broken down into three, not necessarily equal, segments, low, medium and high harm cases $(L, M, H)$, ordered by the size of anticompetitive harm. The purpose is to formalise selection bias and establish what other information is necessary in order to derive an unbiased estimate.

Similarly to Eq.(1), the ratio of sampled harm in each of the three segments has two constituents, non-deterrence and detection:

$$
\lambda_{i}=\left(1-\omega_{i}\right) \sigma_{i}
$$

where $i=L, M, H$.

Assumption 1 With this classification we assume for simplicity that sampled harm can vary across the three segments but not within each segment.

The lower tail of least harmful cases accounts for a proportion $P_{L}$ of the population, and proportion $H_{L}$ of population harm; the upper tail for $P_{H}$ and $H_{H}$, and the middle segment for $P_{M}=\left(1-P_{H}-P_{L}\right)$ and $H_{M}=\left(1-H_{H}-H_{L}\right)$. This is depicted in Figure 2 using a traditional Lorenz curve, in which the population is ranked in ascending order of size (here, harm) along the horizontal axis, and the curve shows the proportion of aggregate harm accounted for by those cases: i.e. how the proportion of the harm accounted for by $x$ proportion of least harmful cases increases with $x .^{13}$

\footnotetext{
${ }^{13}$ The Lorenz curve is non-parametric, but often proves helpful in the analysis of positively skewed
} 
Figure 1: Lorenz curve with three segments of population

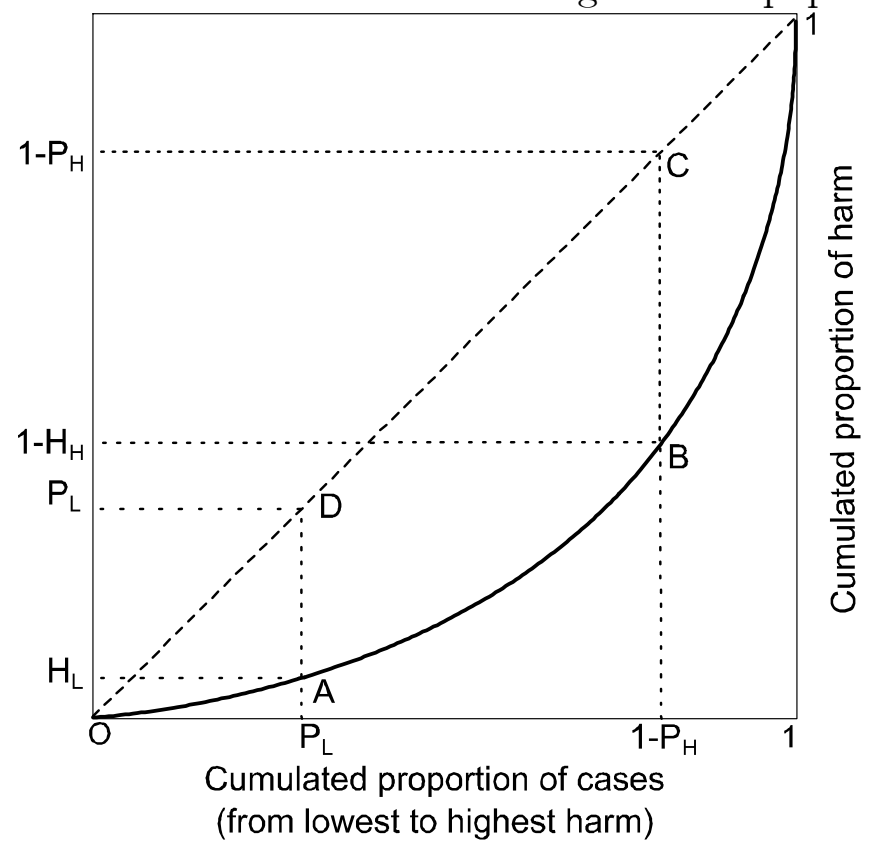

Both axes are therefore normalised to the interval [0,1], and the 45 degree diagonal provides the symmetric benchmark where all cases involve identical harm. The curve must be concave to the diagonal because cases are ranked in ascending order of size, and lies further below the diagonal the more asymmetric is the size distribution.

Assume that each segment is sampled, but with different sample proportions $\lambda_{i}$ $(i=L, M, H)$, thus $\lambda=\sum_{i} \lambda_{i} P_{i}{ }^{14}$

Proposition 1 With random sampling across segments, the simple multiplier $\left(H^{S} / \lambda\right)$ is an unbiased estimator of aggregate population harm $H$. With differential sampling, it is typically biased; the direction and magnitude of bias depends on (i) the sampling

distributions. Traditionally, 'size' might be personal income in studies of income distribution or firm size in studies of industrial concentration; see Lorenz (1905), or Gastwirth (1972).

${ }^{14}$ Thus continuous relationships between the sampling rate and case harm are approximated with a simple three-step function. Although analytically crude, this is sufficiently flexible for present purposes - the relative magnitudes of the $\lambda_{i}$ can capture monotonicity or not, and concavity, linearity or convexity. As explained below, the magnitudes of the $\lambda_{i}$ are sensitive to how broadly defined are the sizes of the different segments $\left(P_{i}\right)$. 
differential and (ii) the relative sizes of mass in the tails.

Proof. Proportionate sample size and the proportionate share of sample harm are:

$$
\begin{aligned}
\lambda & \equiv \lambda_{L} P_{L}+\lambda_{M} P_{M}+\lambda_{H} P_{H} \text { and since } P_{L}+P_{M}+P_{H}=1 \\
\lambda & =\lambda_{M}+\left(\lambda_{L}-\lambda_{M}\right) P_{L}+\left(\lambda_{H}-\lambda_{M}\right) P_{H} .
\end{aligned}
$$

Similarly

$$
H^{S} / H=\lambda_{M}+\left(\lambda_{L}-\lambda_{M}\right) H_{L}+\left(\lambda_{H}-\lambda_{M}\right) H_{H}
$$

From simple manipulation,

$$
H=\frac{H^{S}}{\left.\lambda+\left(\lambda_{M}-\lambda_{L}\right)\left(P_{L}-H_{L}\right)-\left(\lambda_{M}-\lambda_{H}\right)\left(H_{H}-P_{H}\right)\right]}
$$

With random sampling $\left(\lambda_{L}=\lambda_{M}=\lambda_{H}\right), H=H^{S} / \lambda^{15}$, but more generally

$$
H^{S} / \lambda \gtreqless H \text { as }\left(\lambda_{M}-\lambda_{L}\right)\left(P_{L}-H_{L}\right) \gtreqless\left(\lambda_{M}-\lambda_{H}\right)\left(H_{H}-P_{H}\right)
$$

The intuition on the sampling differential is obvious: for a given population distribution $\left(P_{L}, H_{L}, P_{H}\right.$ and $\left.H_{H}\right)$, upward bias is more likely the larger is sampling of the upper tail $\left(\lambda_{H}\right)$ relative to sampling of the lower tail $\left(\lambda_{L}\right)$. Intuition on $\left(P_{L}, H_{L}, P_{H}\right.$ and $\left.H_{H}\right)$ is aided by referring to the Lorenz curve, in which $\left(P_{L}-H_{L}\right)=$ $A D$ and $\left(H_{H}-P_{H}\right)=B C$. Thus

$$
H^{S} / \lambda \gtreqless H \text { as } A D / B C \gtreqless\left(\lambda_{M}-\lambda_{H}\right) /\left(\lambda_{M}-\lambda_{L}\right)
$$

The magnitudes of $\mathrm{AD}$ and $\mathrm{BC}$ reflect the relative sizes of the tails and the extent of population asymmetry, represented by the distance of the Lorenz curve from the

\footnotetext{
${ }^{15}$ In conventional notation, for a sample $n$ with sample mean $x$, drawn from a population $N$ with mean $\mu$, the magnitudes of sample and population harms are $n x$ and $N \mu$ respectively. If the sample is random, $E(x)=\mu$, the ratio of the magnitude of sample harm to proportionate sample size has $E(n x) /(n / N)=N \mu$.
} 
diagonal, but the direction of bias also depends on the precise shape of the Lorenz curve, and therefore the form of the underlying population distribution. To establish the form of this distribution for the purposes of this paper we rely on the empirical distribution of cartel overcharges as explained in Section 5 .

\section{Sample selection}

This section explores the likely relative magnitudes of the sampling proportions $\lambda_{L}$, $\lambda_{M}$ and $\lambda_{H}$. Implications are organised in the form of remarks. For this exercise we use cartel overcharge ${ }^{16}$ as a proxy for harm and turn to the extensive cartel overcharge dataset in Connor (2014) which contains 1504 overcharge observations, 1145 of these occured under regimes when cartels were illegal and 359 when they were legal. Figure 2 compares cartel overcharge densities between these two groups of cases. ${ }^{17}$

In comparing these two types of enforcement regimes we can assume that where cartels are legal there should be no deterrent effect (at least not caused by enforcement) and we also make the implicit assumption that in this case all cartels are equally likely to be detected. In contrast, when cartels are illegal, some cartel harm will be deterred and undeterred harm is not all detected. Therefore comparing the density of overcharge for these two regimes should tell us whether deterrence and detection are higher/lower for given levels of harm. Using this intuition together with the overcharge data, we can make some remarks on the characteristics of undeterred and detected (sampled) harm in the period where cartels are illegal.

Remark 2 If cartels are illegal, harm is most likely to be sampled in the middle segment: $\lambda_{L}<\lambda_{M}>\lambda_{H} \cdot{ }^{18}$

\footnotetext{
${ }^{16}$ Ideally, we would measure harm in terms of consumer welfare loss, and overcharge is admittedly, at best, an approximate proxy. However, there is, of course no equivalent metastudy of previous estimates of cartel harm in terms of lost surplus.

${ }^{17}$ For presentational reasons densities are only plotted up to the 90th percentile of the sample (the top 10\% of the sample ranges from around 70 to 1800, making it difficult to include in a single scale plot).

${ }^{18}$ This is confirmed by formal tests in a short note by Davies and Ormosi (2014).
} 
Figure 2: Density plot of overcharge under illegal (deterrence) and legal (no deterrence) regimes

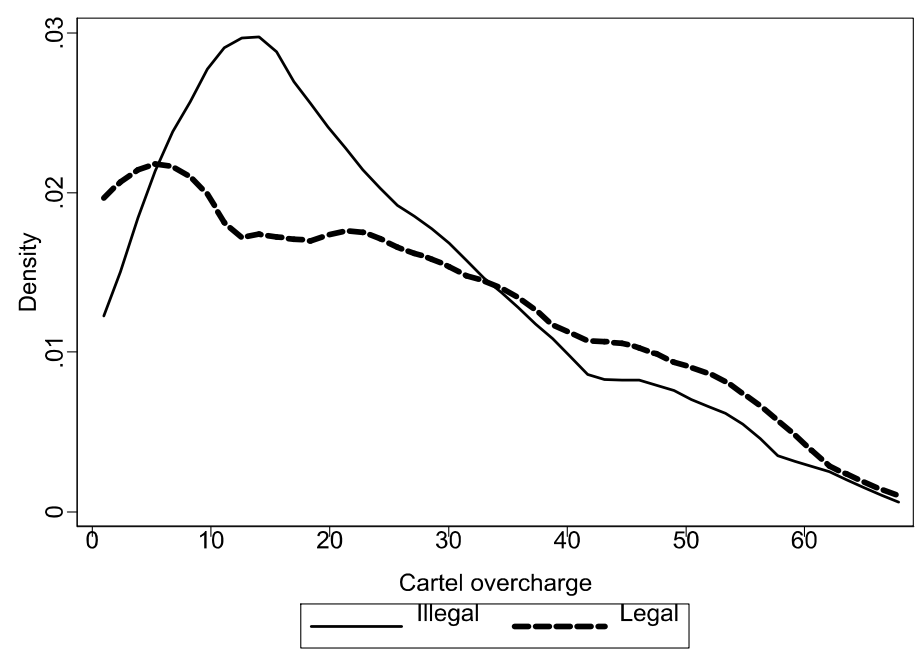

This might be either because low and high harm cases are less likely to be detected, and/or because these cases are more likely deterred. To disaggregate into two effects of detection and deterrence we consider first how detection probability varies with harm. ${ }^{19}$

Anti-cartel enforcement has two arms: leniency applications and 'ex officio' detection (our short-hand for non-leniency cases provoked by consumer complaints or the CA's own monitoring independent of leniency). The existing literature includes some early papers which consider only ex-officio, but more recently the focus has been on the likely impact of leniency programmes. Our detection rate variable can be thought of as the weighted average of these two forms of detection, with the larger weight attached to leniency since this dominates in most jurisdictions.

Most of the recent theoretical and experimental literatures on leniency, such as Chang and Harrington (2012), Jensen and Sørgard (2014), Fonseca and Normann (2012), and Bigoni et al. (2012), suggest that the probability of leniency applications increases with harm. This is because in markets where cartels cause large harm there

\footnotetext{
${ }^{19}$ One could also examine deterrence but naturally more information is available on detection.
} 
is also a larger incentive to deviate from collusion, and less stable cartels are more likely to apply for leniency.

On the other hand, to our knowledge, there is no empirical or experimental evidence to suggest how, if at all, ex-officio detection is related to case harm. Block et al. (1981)'s model assumes that it increases with the cartel markup (harm) because higher prices are more likely to be spotted by customers or the CA, but more recently, there is a growing understanding that it is price fluctuations, rather than levels, that create suspicions in the minds of customers and the CA (Harrington (2005), Harrington (2004)). Employing these findings from previous literature, and recalling the fact that leniency cases typically outnumber ex officio ones, we make the following remarks:

Remark 3 The probability of cartel detection is non-decreasing with case harm: $\sigma_{L} \leq$ $\sigma_{M} \leq \sigma_{H}$

Turning to deterrence, to our knowledge there is no comparable literature on how deterrence varies with harm. However, combining Remarks 2 and 3 allows the following deductions to be made about deterrence:

Remark 4 If detection increases with harm it must follow that the probability of cartel deterrence is highest for higher harms $\omega_{L, M}<\omega_{H}$, although we cannot tell whether $\omega_{L}<,>$, or $=\omega_{M}$.

Remark 5 If detection is invariant with harm it must follow that $\omega_{L}>\omega_{M}<\omega_{H}$, although we cannot tell whether $\omega_{L}<,>$, or $=\omega_{H} \cdot{ }^{20}$

\section{Estimating deterred and undetected harm}

Based on Remarks 3-5, this section applies Eq.(4) to simulate the magnitudes of total potential, undetected and deterred harms. First, total potential harm is estimated

\footnotetext{
${ }^{20}$ In Section 5 we present simulation results also assuming the third possibility: detection rate decreasing with harm. However, we found no previous literature to back up this scenario.
} 
using Eq.(4), with $\lambda:=(1-\omega) \sigma$, but Eq.(4) can also be used to estimate undeterred harm if we re-define the population so as to include only all undeterred cases. In this case $H$ in Eq.(4) is interpreted as population of undeterred harm, and the sample proportions are the proportions of undeterred harm which is detected, $\lambda:=\sigma$. Finally, given estimates of total and undeterred harms, deterred harm is derived as the residual, and in turn, undetected harm as the residual undeterred harm which is not detected. For reference, Appendix A shows the decomposition of total population harm into detected, undetected and deterred harms.

\subsection{Monte Carlo experiments}

To run the simulations using Eq.(4) we need to calibrate (1) the asymmetry in the harm distribution $\left(P_{L, H}\right.$ and $\left.H_{L, H}\right),(2)$ the aggregate deterrence and detection rates ( $\omega$ and $\sigma$ ), and (3) how these rates vary with cartel harm $\left(\omega_{L}, \sigma_{L}, \omega_{H}, \sigma_{H}\right.$ and thus $\left.\lambda_{L}, \lambda_{H}\right)$. To do this, when possible, we call on previous empirical evidence but acknowledging the inevitable uncertainty about how the detection and deterrence rates vary with harm. The Monte Carlo method allows these parameters to assume a wide range of values.

\subsubsection{The population distribution}

Instead of relying on a parametric assumption for the harm distribution we use Connor's (2014) overcharge data to define the three segments $L, M$, and $H{ }^{21}$ The two points of intersection between the legal and illegal regime distribution in Figure 2 provide a natural way to identify the three segments. These occur at overcharge rates of $6 \%$ and $34 \%$. So the lower tail is defined as overcharges less than $6 \%$; this accounts for $15 \%$ of the sample but only $1 \%$ of aggregate harm. The upper tail, overcharge about $34 \%$, accounts for $35 \%$ of the sample and $78 \%$ of the harm. Although this precise choice of magnitudes for each segment of harm is necessarily arbitrary, it

\footnotetext{
${ }^{21}$ In previous versions of this paper we used parametric assumptions of lognormal and Pareto, however we believe the the empirical distribution from Connor's dataset gives a better approximation to the real distribution of cartel harm.
} 
does give a real, evidence-based meaning to low, medium, and high in terms of the likelihood of sampling. ${ }^{22}$

\subsubsection{The aggregate detection and deterrence rates}

We model both $\omega$ and $\sigma$ as random variables. In the case of $\sigma$, there is a fairly large existing literature attempting to estimate the cartel detection rate. Lande and Connor (2012) report the results of 25 previous econometric studies, which yield estimates within the range 0.1 to 0.33 . We therefore specify $\sigma$ here as a random variable with a uniform distribution bounded by this range: $\sigma \sim U(0.1,0.33)$.

For $\omega$, the deterrence rate, there is much less to go on from the previous literature. Therefore, we shall not rule out any possible value between 0 and 1 . Nevertheless, we shall assume relatively smaller probabilities of extreme values (implying virtually no or complete total deterrence). Thus, we assume a symmetric distribution within these bounds, as described by a beta distribution $\omega \sim \operatorname{Bet} a(\alpha, \beta)$, where $\alpha=\beta=2$. This implies mode and mean of $\omega=0.5$, but with non-trivial probabilities of all other values between 0 and 1 . In passing, we note that $\omega=0.5$ is consistent with the most widely cited statistic in the existing literature, from the Deloitte survey study described earlier, based on the opinions of competition lawyers and economists that, for every cartel that occurs, there are another 5 that do not, because of fears of prosecution under competition law. ${ }^{23}$ Note however that, in interpreting our results below, we shall not place undue emphasis on this particular mean value, instead we consider all feasible alternatives.

\subsubsection{The disaggregated sampling rates}

Following Section 4 we distinguish between two cases, based on the alternative assumptions on how detection varies with harm:

\footnotetext{
${ }^{22}$ We ran simulations where the size of the tails and the corresponding proportion of harm were allowed to be random but as long as smaller values were equally likely as higher values the estimates remained in the same ballpark.

${ }^{23}$ The proportion of detected harm is $(1-\omega) \sigma$, then if deterrence is 5 times greater than detection, $\omega=5 \sigma(1-\omega)$. The Deloitte survey also reports that only 1 in 5 cartels are detected, i.e. $\sigma=0.2$. If so, $\omega=0.5$.
} 


\section{Detection rate increases with harm (Remark 4):}

a) Rate of detection: To ensure increasing detection rates across the segments, we take three numbers from the distribution $U(0.1,0.33)$, rank them in ascending order, and assign them to $\sigma_{L}, \sigma_{M}$, and $\sigma_{H}$ in this order, which ensures that $\sigma_{L} \leq \sigma_{M} \leq \sigma_{H}$

b) Rate of deterrence: First we draw three numbers from the distribution $\operatorname{Beta}(2,2)$ and then rank them in ascending order. Because deterrence in this case is assumed to be highest for high harm cases, we assign the greatest to $\omega_{H}$, and decide with a fair draw whether $\omega_{L}$ or $\omega_{H}$ is smaller. This ensures that $\omega_{H}>\max \left(\omega_{L}, \omega_{M}\right)$ in every draw, whilst allowing for all possible values of deterrence.

\section{Detection is invariant with harm (Remark 5):}

a) Rate of detection: Here, trivially, each draw of aggregate $\sigma$ from $\sigma \sim U(0.1,0.33)$ implies the same value for each segment, $\sigma=\sigma_{L}=\sigma_{M}=\sigma_{H}$.

b) Rate of deterrence: Following Remark 5, if $\sigma$ is constant across segments then the lowest and the highest harm cases are more likely deterred. To simulate this in each case we generate three numbers from a beta distribution, $\operatorname{Beta}(2,2)$, and rank them in ascending order. We then set $\omega_{M}$ as the lowest value, and decide randomly with a fair draw whether $\omega_{L}$ or $\omega_{H}$ is larger. This ensures that $\omega_{M}<\min \left(\omega_{L}, \omega_{H}\right)$ for every draw, according to Remark 5, whilst allowing for all possible values of deterrence.

\subsection{Results}

Table 1 reports summary statistics for the simulations, resulting from 10,000 draws. The partitions of the table refer to the alternative assumptions about how the detection rate varies with harm: the first is for detection increasing with harm - the most plausible, following the arguments of Section 4; in the second it is assumed invariant 
with harm, and for completeness, the third assumes detection decreases with harm (although, as we have already argued, there is no theoretical or empirical support for this). In the main, we focus on the results assuming increasing detection.

Table 1 reports the mean simulated values of total potential harm, which is then disaggregated into deterred and undetected harms. Detected harm is normalised as $H^{S}=1$, therefore the harms can be interpreted as multipliers of observed (detected) harm.

These mean values can be interpreted as point estimates, but because of the inevitable uncertainties about our calibrated values, especially for $\omega$, the table also reports $p 5$ and $p 95$. In effect, these set a $90 \%$ confidence interval, in the sense that $90 \%$ of our experiments generate estimates within this range, and $p 5$ and $p 95$ provide conservative upper and lower bounds. Table 1 also reports the values of $\omega$ and $\sigma$ associated with these bounds: the lower bound relates to low aggregate deterrence and detection rates $(0.28$ and 0.16$)$ and the upper bound to a much higher rates (0.75 and 0.29 respectively).

Result 1 According to the point (mean) estimate, total potential anticompetitive harm from cartels is 13.7 larger than the harm detected and observed by the CA. Within this, deterred harm is 9.5 times higher and undetected harm over 3 times greater than the CA's detected harm.

These are clearly very large multipliers, confirming that detected cartels are only the tip of an iceberg of potential cartel harm, most of which is unobserved, either because they are not detected or mainly because they are deterred.

However, as already stressed, these are only point estimates, and more cautiously, we should acknowledge the inevitable uncertainties about the magnitudes of the key deterrence and detection rates - as reflected in the estimated bounds.

Result 2 In terms of the 90 percent bounds, the total harm lies in a range of between 5.9 and 28.9 times greater than the observed harm, deterred harm between 2.2 and 24.1 times greater, and undetected harm between 2.2 and 4.9 greater - in each case, depending on the strength of deterrence and detection. 
Table 1: Monte Carlo results

\begin{tabular}{|c|c|c|c|c|}
\hline variable & mean & median & p5 & p95 \\
\hline \multicolumn{5}{|c|}{ INCREASING DETECTION RATE } \\
\hline total harm & 13.655 & 11.387 & 5.855 & 28.878 \\
\hline deterred harm & 9.506 & 7.276 & 2.247 & 24.143 \\
\hline undetected harm & 3.149 & 2.921 & 2.227 & 4.875 \\
\hline$\omega$ & 0.504 & 0.499 & 0.276 & 0.748 \\
\hline$\omega_{L}$ & 0.403 & 0.396 & 0.112 & 0.723 \\
\hline$\omega_{M}$ & 0.403 & 0.395 & 0.112 & 0.725 \\
\hline$\omega_{H}$ & 0.692 & 0.708 & 0.407 & 0.924 \\
\hline$\sigma$ & 0.227 & 0.228 & 0.157 & 0.292 \\
\hline$\sigma_{L}$ & 0.158 & 0.147 & 0.104 & 0.246 \\
\hline$\sigma_{M}$ & 0.215 & 0.216 & 0.131 & 0.299 \\
\hline$\sigma_{H}$ & 0.273 & 0.282 & 0.185 & 0.326 \\
\hline \multicolumn{5}{|c|}{ CONSTANT DETECTION RATE } \\
\hline total harm & 12.990 & 10.917 & 5.270 & 27.742 \\
\hline deterred harm & 7.783 & 5.759 & 1.496 & 20.641 \\
\hline undetected harm & 4.207 & 3.673 & 2.141 & 7.968 \\
\hline$\omega$ & 0.451 & 0.453 & 0.229 & 0.678 \\
\hline$\omega_{L}$ & 0.595 & 0.605 & 0.276 & 0.888 \\
\hline$\omega_{M}$ & 0.307 & 0.292 & 0.076 & 0.581 \\
\hline$\omega_{H}$ & 0.596 & 0.603 & 0.280 & 0.888 \\
\hline$\sigma$ & 0.214 & 0.214 & 0.112 & 0.318 \\
\hline$\sigma_{L}$ & 0.214 & 0.214 & 0.112 & 0.318 \\
\hline$\sigma_{M}$ & 0.214 & 0.214 & 0.112 & 0.318 \\
\hline$\sigma_{H}$ & 0.214 & 0.214 & 0.112 & 0.318 \\
\hline \multicolumn{5}{|c|}{ DECREASING DETECTION RATE } \\
\hline total harm & 11.709 & 10.348 & 5.763 & 21.864 \\
\hline deterred harm & 5.495 & 3.976 & 0.900 & 14.949 \\
\hline undetected harm & 5.214 & 5.172 & 2.967 & 7.657 \\
\hline$\omega$ & 0.445 & 0.439 & 0.200 & 0.713 \\
\hline$\omega_{L}$ & 0.691 & 0.707 & 0.410 & 0.919 \\
\hline$\omega_{M}$ & 0.401 & 0.394 & 0.106 & 0.724 \\
\hline$\omega_{H}$ & 0.403 & 0.397 & 0.108 & 0.724 \\
\hline$\sigma$ & 0.203 & 0.202 & 0.138 & 0.273 \\
\hline$\sigma_{L}$ & 0.272 & 0.282 & 0.184 & 0.326 \\
\hline$\sigma_{M}$ & 0.215 & 0.215 & 0.131 & 0.298 \\
\hline$\sigma_{H}$ & 0.157 & 0.147 & 0.104 & 0.245 \\
\hline
\end{tabular}


Although these ranges are wide, especially for deterrence, even focussing on the most cautious lower bounds, three key conclusions remain robust: (i) detected harm is only a small fraction (at most one sixth) of total potential harm - the tip of the iceberg; (ii) deterrence is at least twice as effective as detection as a means for removing harm; (iii) undetected harm is at least twice as large as detected harm. ${ }^{24}$

Table 2 develops the implications for evaluating the efficacy of policy in terms of the relative impacts of detection and deterrence. Consider the estimated mean total harm column: if total harm is 13.7 greater than detected harm, then the latter is $7.3 \%$ of total harm. Similarly, a deterrence multiplier of 9.51 implies that 9.51/13.7 = $69.4 \%$ of total harm is deterred. This implies the CA 'removes' $76.7 \%$ of all potential harm, the remainder being undetected. The $p 5$ and $p 95$ columns are constructed similarly.

Table 2: Assessing the 'success' of competition policy

\begin{tabular}{lccc} 
& mean & p5 & p95 \\
Total potential harm & 13.7 & 5.86 & 28.9 \\
\hline detected & 1 & 1 & 1 \\
& $7.30 \%$ & $17.10 \%$ & $3.50 \%$ \\
\hline deterred & 9.51 & 2.25 & 24.1 \\
& $69.40 \%$ & $38.40 \%$ & $83.40 \%$ \\
\hline Success (detected+ deterred) & $76.70 \%$ & $55.50 \%$ & $86.90 \%$
\end{tabular}

Now interpret the lower bound as describing a 'poorly' performing CA (recall that at the lower bound, aggregate deterrence and detection rates are relatively low at 0.28 and 0.16 respectively); and the upper bound as a 'good' CA (much higher rates of 0.75 and 0.29 respectively). The table then provides a quantification of poor, good and average. Thus the mean CA is successful at 'removing' three quarters of all potential cartel harm, mainly by deterrence, the poor CA removes little more than half, while the good CA removes nearly $90 \%$.

\footnotetext{
${ }^{24}$ Another implication is that the treble damages rules applied in cartel cases are good approximation if the purpose of the rule is to redistribute wealth from undetected harm (one function of treble damages rules is to account for undetected infringements). They are not adequate as a deterrent though as detection probabilities are estimated to be under $1 / 3$.
} 
Table 2 also reveals what at first sight appears to be paradoxical. Note that the poor CA actually detects a greater percentage of potential harm - in spite of having a lower detection rate. However, there is no paradox - under a poor CA regime, far less harm is deterred and so many more cases actually occur, and this numbers effect outweighs the lower rate. ${ }^{25}$

Result 3 These estimates suggest that CAs 'remove' between half and nearly ninetenths of all potential harm. A corollary of this is that it can be misleading to judge the performance of a CA merely from the magnitude of harm it detects. While higher detected harm can signal a well-performing CA, it might also occur when a $C A$ is particularly ineffectual at deterring harmful cartels - more detected harm might reflect less effective deterrence.

It is important to emphasise that because of the simplification of working with an artificial trichotomy of anti-competitive harm (low, medium, high) the estimates above should only be considered as an approximation of the real magnitudes of harm.

Finally, the above results all rely on the assumption that the detection rate increases with harm. If we now return to Table 1 , it appears that the above conclusions are substantively robust to assuming instead a constant detection rate: (i) for total harm, both the mean and lower bound estimates fall very slightly (by less than one point); (ii) for undetected harm, the mean and range between bounds both increase slightly; and (iii) for deterred harm, both the mean and lower bound decrease, but in all cases the magnitude of changes is very small. ${ }^{26}$

\subsection{The simple multiplier}

Part of our motivation for this paper was to illustrate how one might tackle the sample selection problem, which is endemic in much of empirical IO. In this case, the bias might occur if one simply uses aggregate estimates of $\omega$ and $\sigma$ and assumes

\footnotetext{
${ }^{25}$ Neven and Zengler (2008) also voiced this concern.

${ }^{26}$ In fact, even employing the assumption of a decreasing detection rate does not substantively change conclusions - notably, the mean multiplier for total harm remains as high as 11.7 and the range is little changed.
} 
random sample selection, to estimate how much deterred and undetected cartel harm there is, given an estimate of detected harm. We referred to this approach as the simple multipliers (see Remark 1 above).

We can now assess this concern as follows. For simplicity, assume fixed values of $\omega=0.5$ and $\sigma=0.215$ - these are the means of the distributions we use in our simulations, and both can be justified by referring to previous empirical literatures, as described earlier. ${ }^{27}$

Using the multipliers as defined in Remark 1 above, these generate the following estimates of harm: Total $=9.3$, Deterred $=4.65$, and Undetected $=3.65$ times the observed harm.

Result 4 Simple multiplier estimates each lie within the 90 percent bounds generated by our simulations, and, with that level of confidence at least, we cannot reject the null of no bias. Nevertheless, for deterred harm the multiplier estimate is 50 percent lower than the mean simulation, while undetected harm is 20 percent larger. In aggregate, total potential harm according to the simple multiplier is one third lower than the simulated mean.

\section{Concluding remarks}

The introduction raised two ambitious questions: how much harm can cartels cause to an economy, and how successful are CAs in rectifying that harm? This paper is the first step towards answering these questions. It proposes a methodology in which the cartels detected by a CA are interpreted as a sample drawn from an otherwise unknown population, which also includes deterred cases and cases which the CA did not detect. This methodology identifies what information is required in order to quantify the magnitudes of the unknown deterred and undetected harms: (i) the nature of the potential population distribution of harm, (ii) the magnitudes of the aggregate probabilities of deterrence and detection, and (iii) how these probabilities vary with case harm.

\footnotetext{
${ }^{27}$ For $\sigma$, this is the midpoint of the estimates reported by Lande and Connor (2012), and for $\omega$ this is the value implied by the Deloitte survey study.
} 
We proceed, where possible, by employing results from previous empirical and theoretical literatures. These provide useful insights on: the nature of the population distribution; a plausible range of magnitudes for the aggregate detection probability; and in which segments of the distribution deterrence and detection are more likely. In the absence of previous empirical works on the likely magnitude of the aggregate deterrence probability, we leave open the possibility of a wide range of potential magnitudes for this parameter in the Monte Carlo simulation. Recognising this element of potential ignorance, when interpreting the results of the simulation experiments, we place considerable emphasis on the bounds to our estimates, as well as the point estimates.

A number of important results emerge. Even if we focus only on the most cautious lower bound estimates, we find that: (i) the harm detected by the CA really is only the tip of the iceberg, accounting for only a small fraction (at most one sixth) of total potential harm; (ii) deterrence is at least twice as effective as detection as a means for removing harm; (iii) undetected harm is at least twice as large as detected harm. Less cautiously, according to the point (mean) estimates, all three effects are much greater.

Some of the implications are self-evident. For policy-makers, deterrence is arguably the most important arm of cartel policy, and harm due to undetected cartels is likely to be considerable. Some are less obvious but important. In particular, measuring the success of a CA simply by calculating the amount of harm it removes by virtue of cartel busts can be misleading. Indeed, we use the results of our simulations to show that a 'poor' CA (relatively ineffectual in deterring and detecting) may actually detect more harm than a 'good' CA, simply because its inability to deter leaves far more cartels out there to be detected.

More academically, another motivation for this paper was to use policy evaluation as an example in which to explore selection bias when much of the population is not observed. On this, the paper suggests a simple but important proposition. In many aspects of economic and social life, positively skewed distributions apply - a small proportion of causes (e.g. 20 per cent) generate a very large proportion of effects (e.g. 80 per cent), e.g. the largest few websites generate the vast majority of internet 
advertising income, the largest customers generate most revenues for businesses, etc. This is well understood in general and here we see that the same is true for cartelinduced harm. If so, even small departures from a random sample can lead to potentially large selection bias. In this context, this implies that the use of 'simple multipliers', which ignore within population variance, can be potentially misleading.

\section{References}

Baker, J. B., 2003. The case for antitrust enforcement. Journal of Economic Perspectives 17 (4), 27-50.

Becker, G. S., 1968. Crime and punishment: An economic approach. The Journal of Political Economy 76 (2), 169-217.

Bigoni, M., Fridolfsson, S.-O., Coq, C. L., Spagnolo, G., 2012. Fines, leniency and rewards in antitrust. RAND Journal of Economics 43 (2), 368-390.

Block, M. K., Nold, F. C., Sidak, J. G., 1981. The deterrent effect of antitrust enforcement. Journal of Political Economy 89 (3), 429-445.

Bryant, P. G., Eckard, E. W., 1991. Price fixing: The probability of getting caught. The Review of Economics and Statistics 73 (3), 531-536.

Chang, M.-H., Harrington, J. E., 2012. The impact of a corporate leniency program on antitrust enforcement and cartelization. mimeo.

Combe, E., Monnier, C., Legal, R., 2008. Cartels: The probability of getting caught in the european union. BEER Paper 12.

Crandall, R. W., Winston, C., 2003. Does antitrust policy improve consumer welfare? assessing the evidence. Journal of Economic Perspectives 17 (4), 3-26.

Davies, S., Ormosi, P., 2014. The deterrent effect of anti-cartel enforcement: A tale of two tails. CCP Working Paper 14 (6). 
Davies, S. W., Ormosi, P. L., 2012. A comparative assessment of methodologies used to evaluate competition policy. Journal of Competition Law and Economics 8 (4), 769-803.

Deloitte, 2007. The deterrent effect of competition enforcement by the OFT. Office of Fair Trading OFT962.

Economics, L., 2011. The impact of competition interventions on compliance and deterrence. Office of Fair Trading OFT1391.

Elzinga, K. G., Breit, W., 1973. Antitrust penalties and attitudes toward risk: An economic analysis. Harvard Law Review 86, 693.

Fonseca, M. A., Normann, H.-T., 2012. Explicit vs. tacit collusion - the impact of communication in oligopoly experiments. European Economic Review 56 (8), $1759-1772$.

Gastwirth, J. L., 1972. The estimation of the lorenz curve and gini index. Review of Economics and Statistics 54 (3), 306-316.

Ginsburg, D. H., Wright, J. D., 2010. Antitrust sanctions. Competition Policy International 6 (2), 3-39.

Harberger, A. C., 1954. Monopoly and resource allocation. The American Economic Review 44 (2), 77-87.

Harrington, J. E., 2004. Cartel pricing dynamics in the presence of an antitrust authority. The RAND Journal of Economics 35 (4), 651-673.

Harrington, J. E., 2005. Optimal cartel pricing in the presence of an antitrust authority. International Economic Review 46 (1), 145-169.

Harrington, J. E., Chang, M.-H., 2012. Endogenous antitrust enforcement in the presence of a corporate leniency program. mimeo. 
Jensen, S., Sørgard, L., 2014. Fine schedule with heterogeneous cartels: Are the wrong cartels deterred? Working Paper.

Katsoulacos, Y., Ulph, D., 2013. Antitrust penalties and the implications of empirical evidence on cartel overcharges. The Economic Journal 123 (572), 558-581.

Kobayashi, B. H., 2001. Antitrust, agency, and amnesty: An economic analysis of the criminal enforcement of the antitrust laws. George Washington Law Review $715,735-739$.

Lande, R. H., Connor, J. M., 2012. Cartels as rational business strategy: Crime pays. Cardozo Law Review 34 (2), 427-490.

Landes, W. M., 1983. Optimal sanctions for antitrust violations. University of Chicago Law Review 50, 652.

Lorenz, M. O., 1905. Methods of measuring the concentration of wealth. Publications of the American Statistical Association 9 (70), 209-219.

Miller, N. H., 2009. Strategic leniency and cartel enforcement. American Economic Review 99 (3), 750-768.

Ormosi, P. L., 2012. Evaluating the impact of competition law enforcement. OECD Working Papers DAF/COMP/WP2(2012)5.

Ormosi, P. L., 2014. A tip of the iceberg? the probability of catching cartels. Journal of Applied Econometrics 29 (4), 549-566.

Schildberg-Hörisch, H., Strassmair, C., 2012. An experimental test of the deterrence hypothesis. Journal of Law, Economics and Organization 28 (3), 447-459.

Werden, G. J., 2008. Assessing the effect of antitrust enforcement in the united states. De Economist 156 (4), 433-451.

Werden, G. J., Hammond, S. D., Barnett, B. A., 2012. Deterrence and detection of cartels: Using all the tools and sanctions. Antitrust Bulletin 56 (2). 


\section{A Decomposition of Population Harm}

Detected Harm as proportion of Population Harm:

$$
\frac{H^{D T}}{H}=\sigma(1-\omega)+\left(H_{H}-P_{H}\right)\left\{\sigma_{H}\left(1-\omega_{H}\right)-\sigma_{L}\left(1-\omega_{L}\right)\right\}
$$

Deterred Harm as proportion of Population Harm:

$$
\frac{H^{D R}}{H}=\omega+\left(H_{H}-P_{H}\right)\left(\omega_{H}-\omega_{L}\right)
$$

Undetected Harm as proportion of Population Harm:

$$
\frac{H^{U D T}}{H}=(1-\sigma)(1-\omega)+\left(H_{H}-P_{H}\right)\left\{\left(1-\sigma_{H}\right)\left(1-\omega_{H}\right)-\left(1-\sigma_{L}\right)\left(1-\omega_{L}\right)\right\}
$$

\section{B An extension: CA errors}

So far, the methodology abstracts away from the possibility of CA errors, either by undesirable deterrence or intervention in pro-competitive cases (Type 1), or failure to intervene in welfare-reducing cases (Type 2). We believe that it is a reasonable approximation for cartels that pro-competitive cartels do not exist, and that the CA always prosecutes a cartel of which it is aware.

However, we are open to the possibility of enforcement error where the CA chooses not to intervene in some cartel cases because it assesses the evidence to be insufficiently strong to rebut any potential appeal (Type 2 error). Harrington and Chang (2012) incorporates the possibility of Type 2 errors in their theoretical treatment of anti-cartel enforcement. ${ }^{28}$ These errors can be built-into our framework by introducing a further conditional probability into Eq.(1). Defining as the random variable $I$ the proportion of intervened harm intervene in a harmful case which is undeterred and detected, then the observed harm:

\footnotetext{
${ }^{28}$ Schinkel and Tuinstra (2004) examine the theoretical possibility of this. The main difference is that their paper focuses on a dynamics of these errors whereas we only propose a snapshot analysis of what one can deduce from the observed to the unobserved.
} 


$$
H_{\sigma}=(1-\omega) \sigma(1-I) H
$$

In this case, $I$ is the probability of Type 2 error. To give an approximate idea of the possible impact, suppose $E(I)=0.1$ and that it is invariant with case harm. Observed sample harm should now be interpreted as the magnitude of intervened detected harm, where this is only $90 \%$ of 'true' detected harm (i.e. detected harm is $1 / 0.9=1.11$ ), of which the CA fails to recognise 0.11 (i.e. $11 \%$ of the intervened harm), due to Type 2 error. Without this adjustment, non-intervened harm is subsumed within our estimates of deterred harm. While the correction would be larger if Type 2 errors are more likely for high harm cartels, this is unlikely to substantively change any of our main conclusions.

\section{Histograms of the simulation results}


Figure 3: Simulated magnitudes of total potential harm
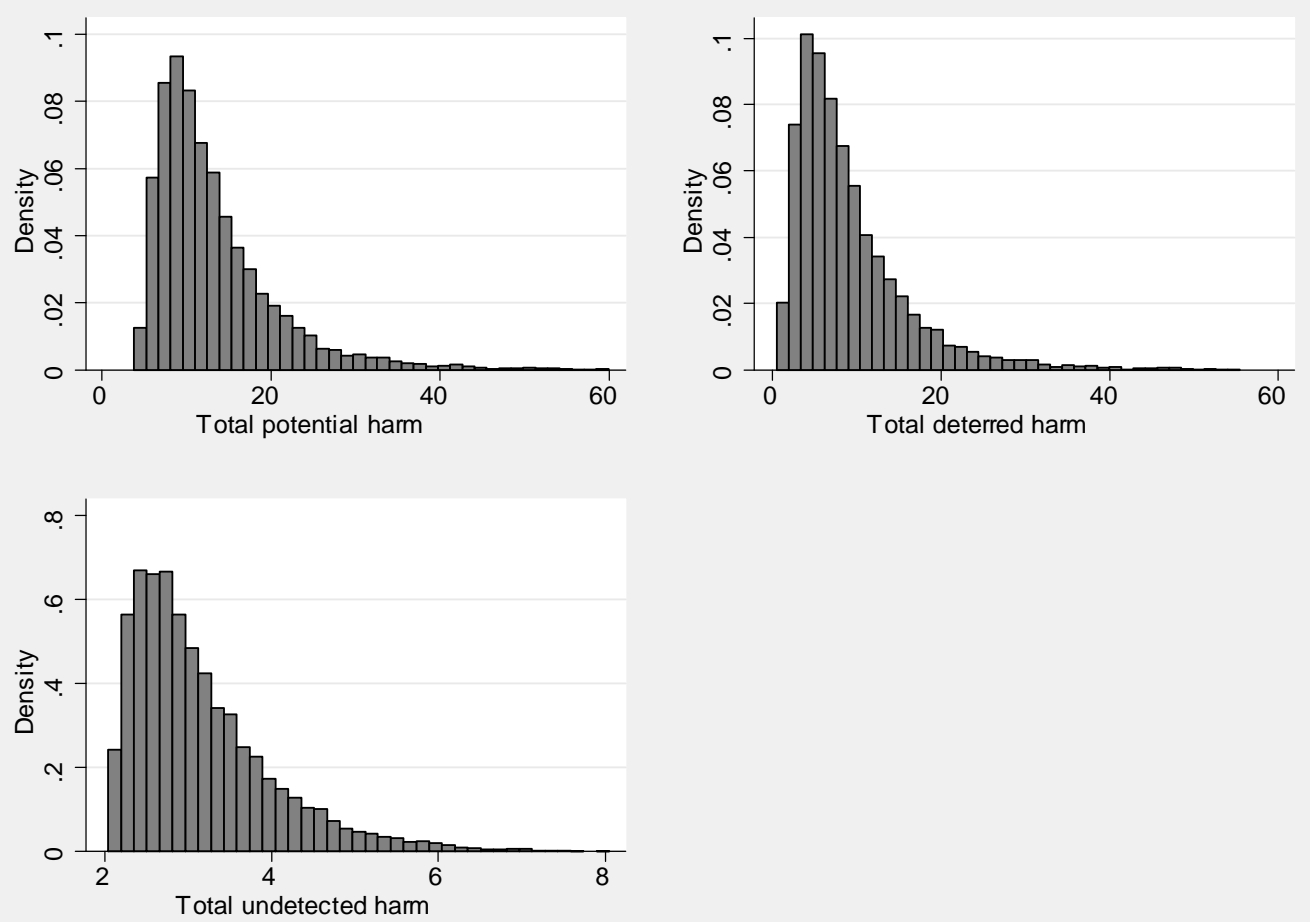